\title{
Associations among systemic blood pressure, microalbuminuria and albuminuria in dogs affected with pituitary- and adrenal-dependent hyperadrenocorticism
}

\author{
Yu-Hsin Lien ${ }^{1,2 \dagger}$, Tsai-Yuan Hsiang ${ }^{2 \dagger}$, Hui-Pi Huang ${ }^{2^{*}}$
}

\begin{abstract}
Background: Hypertension and proteinuria are medical complications associated with the multisystemic effects of long-term hypercortisolism in dogs with hyperadrenocorticism (HAC).

Methods: This study investigated the relationships among adrenocorticotropic hormone (ACTH)-stimulation test results, systemic blood pressure, and microalbuminuria in clinically-healthy dogs $(n=100)$, in dogs affected with naturally occurring pituitary-dependent $(\mathrm{PDH} ; \mathrm{n}=40)$, or adrenal-dependent hyperadrenocorticism $(\mathrm{ADH} ; \mathrm{n}=30)$.

Results: Mean systemic blood pressure was similar between clinically healthy dogs and dogs with HAC ( $p=0.803)$. However the incidence of hypertension was highest in dogs with ADH $(p=0.017)$, followed by dogs with PDH, with the lowest levels in clinically healthy dogs $(p=0.019)$. Presence of microalbuminuria and albuminuria in clinically healthy dogs and dogs affected with HAC was significantly different $(p<0.001)$; incidences of albuminuria followed the same pattern of hypertension; highest incidence in dogs with $\mathrm{ADH}$, and lowest level in clinically healthy dogs; but microalbuminuria showed a different pattern: clinically healthy dogs had highest incidences and dogs with ADH had lowest incidence. The presence of albuminuria was not associated with blood pressure values, regardless of whether dogs were clinically healthy or affected with $\mathrm{ADH}$ or PDH ( $p=0.306)$.

Conclusions: Higher incidence of hypertension and albuminuria, not microalbuminuria was seen in dogs affected with HAC compared to clinically healthy dogs; incidence of hypertension and albuminuria was significantly higher in dogs affected with ADH compared to PDH. However, presence of albuminuria was not correlated with systemic blood pressure.
\end{abstract}

\section{Background}

Hyperadrenocorticism (HAC, Cushing's syndrome) is a common endocrine disorder in dogs and is characterized by chronically elevated circulating concentrations of the steroid hormones produced by the adrenal cortex (e.g. cortisol) [1]. The multi-systemic effects of longterm hypercortisolism in dogs results in a variety of medical complications such as hypertension and proteinuria [1-4]. Hypertension develops by several mechanisms, including excessive renin concentrations which

\footnotetext{
* Correspondence: hphuang@ntu.edu.tw

† Contributed equally

${ }^{2}$ Institute of Clinical Veterinary Science, National Taiwan University, No. 1,

Section 4, Roosevelt Road, Taipei 100, Taiwan

Full list of author information is available at the end of the article
}

lead to activation of the renin-angiotensin system, increased vascular sensitivity to catecholamine, and increased concentrations of aldosterone [5-11]. These conditions warrant both prompt, in-depth evaluation of organ damage and treatment. For example, glomerular damage secondary to hypertension may further result in albuminuria which is associated with deteriorated renal function [12-17].

Apart from hypertension, many complications associated with Cushing's syndrome in humans are also linked with increased urinary albumin excretion rate. These include dyslepidaemia, reduced insulin sensitivity, and hyper-coagulative status. These complications are also associated with canine HAC $[1,16,18,19]$. 
Microalbuminuria is defined as the presence of a small amount of albumin ( 1 to $30 \mathrm{mg} / \mathrm{dL}$ ) in the urine [16]. Microalbuminuria assays are more sensitive to albumin loss in the urine than the common urine dipstick, and urinary protein:creatinine ratio tests can detect protein loss through the urine with lower limits-of-detection than urine dipsticks. Microalbuminuria is used as an indicator in screening for early-stage renal and cardiovascular diseases in humans $[12,14,17,20,21]$. It is a well-known risk factor associated with deteriorated cardiovascular mortality and diabetic nephropathy in human beings [12-14,17]. Similarly, microalbuminuria is associated with renal and systemic sub-clinical diseases in dogs and cats [22-24].

The purposes of this study were to report the relationship between ACTH-stimulation tests, systemic blood pressure, microalbuminuria and albuminuria in dogs affected with naturally occurring pituitary-dependent $(\mathrm{PDH})$ and adrenal-dependent hyperadrenocorticism $(\mathrm{ADH})$.

\section{Methods}

Animals

Informed consent was obtained from all dog owners prior to the study, and number of animals of each breed in each group are shown in Table 1.

\section{Clinically healthy dogs}

One hundred clinically healthy client-owned dogs visiting the National Taiwan University Veterinary Hospital from 2005 to 2009 for routine health checkups and/or annual vaccination were included in this study. Of the clinically healthy dogs, 29 were intact females, 19 were spayed females, 45 were intact males, and 7 were castrated males. The average age was $5.1 \pm 3.5$ years (range 0.9 to 15 years), and average body weight was $11.6 \pm 9.1 \mathrm{~kg}$ (range 1.8 to $35.0 \mathrm{~kg}$ ). Thorough physical examination, blood pressure measurements and routine blood work (complete blood counts and biochemical profiles) were performed to exclude heart and renal disorders that might affect blood pressure or cause microalbuminuria or albuminuria.

\section{Dogs affected with hyperadrenocorticism}

Medical records of 278 dogs in the National Taiwan University Veterinary Hospital during 2005-2009 with clinical signs of HAC and results of ACTH stimulation tests consistent with hyperadrenocorticism were screened. Only 70 of these records included conclusive results from an ACTH stimulation test, ultrasonographic evidence of adrenal glands, blood pressure measurements and microalbuminuria analysis. Therefore, only 70 dogs were included in the study.

Inclusion criteria of HAC were clinical signs consistent with a diagnosis of HAC (e.g., polydipsia, polyuria, polyphagia, decreased activity, panting, a potbellied
Table 1 Breeds and numbers of the 100 clinical healthy dogs, 40 dogs affected with pituitary-dependent hyperadrenocorticism (PDH) and 30 dogs affected with adrenal-dependent hyperadrenocorticism (ADH)

\begin{tabular}{|c|c|c|c|}
\hline Breed & Clinically Healthy & PDH & $\mathrm{ADH}$ \\
\hline Akita inu & 0 & 0 & 1 \\
\hline Beagle & 1 & 2 & 1 \\
\hline Bichon frise & 0 & 1 & 0 \\
\hline Chihuahua & 0 & 1 & 0 \\
\hline Cocker Spaniel & 2 & 1 & 1 \\
\hline Japanese spitz & 0 & 2 & 1 \\
\hline Golden retriever & 2 & 0 & 0 \\
\hline Lhasa apso & 1 & 0 & 0 \\
\hline Maltese terrier & 20 & 7 & 9 \\
\hline Miniature pinscher & 3 & 1 & 1 \\
\hline Miniature poodle & 10 & 3 & 1 \\
\hline Miniature schnauzer & 5 & 2 & 2 \\
\hline Mixed breed & 22 & 7 & 2 \\
\hline Papillon & 1 & 0 & 0 \\
\hline Pekingese & 1 & 0 & 0 \\
\hline Pomeranian & 9 & 3 & 3 \\
\hline Pug & 1 & 0 & 0 \\
\hline Shetland sheep dog & 1 & 0 & 0 \\
\hline Shihba inu & 0 & 1 & 0 \\
\hline Shih Tzu & 5 & 4 & 5 \\
\hline Short-haired dachshund & 0 & 0 & 1 \\
\hline West Highland white terrier & 0 & 1 & 0 \\
\hline Yorkshire Terrier & 16 & 4 & 2 \\
\hline Total & 100 & 40 & 30 \\
\hline
\end{tabular}

appearance, and dermatologic problems), results of routine serum biochemical analyses that were consistent with a diagnosis of HAC (i.e., elevated activities of hepatic enzymes), and affirmative results of adrenocorticotropic hormone $(\mathrm{ACTH})$ stimulation tests (post-ACTH cortisol concentration $>20 \mu \mathrm{g} / \mathrm{dL}$ ) [25]. Dogs with inconclusive results of $\mathrm{ACTH}$ stimulation tests were excluded from the study. Further differentiation between $\mathrm{PDH}$ or ADH was based upon adrenal ultrasonographic findings $[1,26,27]$. Size, shape, presence of nodular appearance and hyperechoic foci: normal or mild enlarged adrenal glands were classified as $\mathrm{PDH}$; whereas adrenal glands with a nodular (or mass) appearance and hyperechoic foci were classified as ADH [1,26,27].

All included dogs were required to have had an ACTH stimulation test with intramuscular injection of $0.25 \mathrm{mg}$ synthetic ACTH (Cortrosyn, Organon, The Netherlands). Informed consent was obtained from all owners prior to diagnostic testing. Blood samples for serum cortisol measurements were collected via cephalic venipuncture immediately before, and 1 hour after synthetic ACTH was injected. Serum cortisol concentrations were measured by a validated radioimmunoassay 
(Coat-A-Count Cortisol, Diagnostic Products Corp., U.S.A.) [27].

In this study, 70 dogs were affected with $\mathrm{HAC}$, with the mean age of $11.7 \pm 2.7$ years and ranged from 4.5 to 18 years. Dogs had a mean body weight of $9.0 \pm 7.6 \mathrm{~kg}$ ranging from 2.1 to $43.4 \mathrm{~kg}$. Twenty-seven were spayed females, 21 were intact males, and 11 each of castrated males and intact females. Among these 70 cases, 40 dogs affected with PDH and 30 dogs affected with ADH (functional adrenal tumor). The mean age of the 40 dogs affected with $\mathrm{PDH}$ was $10.7 \pm 2.7$ years, ranging from 4.5 to 16 years with a mean body weight of $9.9 \pm$ $8.6 \mathrm{~kg}$, ranging from 2.1 to $43.4 \mathrm{~kg}$. Sixteen were spayed females, 6 were intact females, 12 were intact males and 6 were castrated males. The mean age of the dogs affected with $\mathrm{ADH}$ was $12.9 \pm 2.2$ years, ranging from 8 to 18 years. This group had a mean body weight of $7.9 \pm 5.6 \mathrm{~kg}$, ranging from 3.0 to $24.5 \mathrm{~kg}$. Eleven were spayed females, 5 were intact females, 9 were intact males, and 5 were castrated males. Breeds of 70 dogs affected with HAC are shown in Table 2.

\section{Systemic blood pressure measurement}

Systemic blood pressure values of 100 clinically healthy dogs and 70 dogs affected with (pre-treated) HAC were evaluated by the same protocol-Doppler sphygmomanometry. A Doppler Ultrasonic Flow Detector (Model 811-B, Parks Medical Electronics Inc., U.S.A.) with an inflatable cuff width of $1.9,2.5,3,4$, or $5 \mathrm{~cm}$ (depending on the circumference of the antebrachium) was used. The cuff was wrapped around the middle part of the antebrachium, and a Doppler probe coated with ultrasonic transmission gel was positioned over the palmar area to detect blood flow from the arteria digitalis palmaris communis. The cuff was then inflated and deflated to obtain a systemic blood pressure reading via an aneroid pressure gauge. During systemic blood pressure measurement, the antebrachium was maintained at the level of the heart. A series of 5 readings (with 10 to
20 seconds between consecutive measurements) was obtained for each dog, and all measurements were completed within 6 minutes. To minimize procedural stress, all dogs were allowed to assume a comfortable position with only gentle restraint by their owners. The dogs remained in the same position throughout systemic blood pressure measurement. The final systemic blood pressure value was calculated as the mean of 5 readings [28]. The heart rate was manually recorded by pulse Doppler ultrasound detection over a period of $20 \mathrm{sec}-$ onds after the systemic blood pressure value was measured. For this study, hypotension, normotension and hypertension were defined as $<100 \mathrm{mmHg}, 100-160$ $\mathrm{mmHg}$, and $>160 \mathrm{mmHg}$, respectively.

\section{Microalbuminuria assay and urinary albumin:creatinine ratio}

Freshly voided urine samples from 100 clinically healthy dogs and 70 dogs affected with (pre-treated) HAC were collected. Urine albumin analysis was carried out within four hours after the freshly voided urine samples were collected. Urine samples with hematuria ( $\geq 10 \mathrm{RBC} /$ high power of field), pyuria ( $\geq 5 \mathrm{WBC} / \mathrm{hpf}$ ), or bacteriuria was excluded after a light-microscopic examination of urine sediment. The urine albumin and creatinine concentrations were semi-quantitated (Clinitek Microalbumin Reagent Strips and Clinitek Status Analyser, Bayer Diagnostic Mfg. Ltd., UK) and urinary albumin:creatinine ratio (UACR) was calculated accordingly. In this study, no microalbuminuria, microalbuminuria and albuminuria were defined as UACR $<30 \mathrm{mg} / \mathrm{g}(0.03), 30-300 \mathrm{mg} / \mathrm{g}$ (0.03-0.3), and $>300 \mathrm{mg} / \mathrm{g}(0.3)$, respectively.

\section{Statistical analysis}

Comparison of systemic blood pressure between clinically healthy dogs and dogs affected with either PDH or ADH were evaluated by means of ANOVA. Relationship between post-ACTH cortisol concentrations and systemic blood pressure was analyzed by use of linear

Table 2 Systemic blood pressure (SBP) and status of urine albumin excretion in clinically healthy dogs and dogs affected with pituitary-dependent hyperadrenocorticism (PDH) or adrenal-dependent hyperadrenocorticism (ADH)

\begin{tabular}{|c|c|c|c|}
\hline & Clinically healthy dogs $(n=100)$ & $\mathrm{PDH}(\mathrm{n}=40)$ & $\mathrm{ADH}(\mathrm{n}=30)$ \\
\hline Mean SBP $(\mathrm{mmHg})$ & $152.8 \pm 27.3$ & $142.2 \pm 24.9$ & $164.1 \pm 36.7 \dagger$ \\
\hline Hypotension \% (n) & $1 \%(1 / 100)$ & 0 & 0 \\
\hline Normotension \% (n) & $87 \%(87 / 100)$ & $80 \%(32 / 40)$ & $53.3 \%(16 / 30)$ \\
\hline Hypertension \% (n) & $12 \%(12 / 100)$ & $20 \%(8 / 40)$ & $46.7 \%(14 / 30)^{*} \dagger$ \\
\hline \multicolumn{4}{|l|}{ Status of urine albumin } \\
\hline No microalbuminuria \% (n) & $53 \%(53 / 100)$ & $15 \%(6 / 40)$ & $10 \%(3 / 30)$ \\
\hline Microalbuminuria \% (n) & $45 \%(45 / 100)$ & $52.5 \%(21 / 40) *$ & $20 \%(6 / 30) *+$ \\
\hline Albuminuria \% (n) & $2 \%(2 / 100)$ & $32.5 \%(13 / 40) *$ & $70 \%(21 / 30) *+$ \\
\hline
\end{tabular}

${ }^{*} p<0.05$ relative to clinically healthy dogs. $\dagger p<0.05$ relative to $\mathrm{PDH}$. 
regression. Association between systemic blood pressure, microalbuminuria status (no microalbuminuria, microalbuminuria and albuminuria) and health status (clinically healthy versus HAC) were evaluated by Pearson's chisquare test. All statistical analyses were performed using commercially available software (SPSS, version 13.0, SSPS Inc, U.S.A.). Continuous data are presented as mean \pm SD. Statistical significance was determined by a $p$ value $<0.05$.

\section{Results}

Systemic blood pressure and presence of microalbuminuria/albuminuria in clinically normal dogs ( $\mathrm{n}$ $=100$ )

The mean systemic pressure of 100 clinically healthy dogs was $152.8 \pm 27.3 \mathrm{mmHg}$. One $(1 \%, 1 / 100)$ was hypotensive, $87 \%(87 / 100)$ were normotensive, and $12 \%$ $(12 / 100)$ were hypertensive. Among the 100 clinically healthy dogs, 53\% (53/100) did not have microalbuminuria, $45 \%(45 / 100)$ had microalbuminuria, and $2 \%$ (2/100) had albuminuria (Table 2$)$.

\section{Systemic blood pressure and microalbuminuria/} albuminuria in dogs affected with HAC $(n=70)$

The mean systemic arterial pressure in dogs affected with HAC was $151.7 \pm 32.1 \mathrm{mmHg}$. None was hypotensive, $68.6 \%(48 / 70)$ were normotensive, and $31.4 \%$ (22/70) were hypertensive. In 70 dogs with HAC, $12.9 \%$ $(9 / 70)$ did not have microalbuminuria, 38.6\% (27/70) had microalbuminuria, and $48.6 \%(34 / 70)$ had albuminuria (Table 2).

Systemic blood pressure, presence of microalbuminuria/ albuminuria and results of ACTH stimulation tests in dogs affected with PDH $(n=40)$

The mean systemic blood pressure in dogs affected with $\mathrm{PDH}$ was $142.2 \pm 24.9 \mathrm{mmHg}$. None was categorized as hypotensive, $80 \%(32 / 40)$ were normotensive, and $20 \%$ (8/40) were hypertensive. Among the 40 dogs affected with PDH, 15\% (6/40) did not have albuminuria, 52.5\% $(21 / 40)$ had microalbuminuria, and $32.5 \%$ (13/40) had albuminuria. Pre- and post-ACTH cortisol concentrations in the dogs affected with PDH were $4.6 \pm 2.9$ (ranging from 1.9 to 14.1 ) and $25.5 \pm 11.6$ (ranging from 21.6-63.1) $\mu \mathrm{g} / \mathrm{dL}$, respectively (Table 2 ).

Systemic blood pressure, presence of microalbuminuria/ albuminuria and results of ACTH stimulation tests in dogs affected with ADH $(n=30)$

The mean systemic blood pressure in dogs affected with $\mathrm{ADH}$ was $164.1 \pm 36.7 \mathrm{mmHg}$. None was hypotensive, $53.3 \%(16 / 30)$ were normotensive, and $46.7 \%(14 / 30)$ were hypertensive. Among the 30 dogs with $\mathrm{ADH}, 10 \%$ (3/30) did not have microalbuminuria, 20\% (6/30) had microalbuminuria, and $70 \%$ had albuminuria. Pre- and post-ACTH cortisol concentrations in dogs affected with $\mathrm{ADH}$ were $8.5 \pm 6.0$ (ranging from 2.9 to 28.7 ) and $33.2 \pm 23.7$ (ranging from 23.6-110.2) $\mu \mathrm{g} / \mathrm{dL}$, respectively (Table 2).

\section{Comparison of systemic blood pressure and presence of} microalbuminuria/albuminuria between clinically healthy dogs and dogs affected with HAC

The mean systemic blood pressure was not different between clinically healthy dogs and dogs with HAC ( $p=$ 0.803 ). However, incidence of hypertension between clinically healthy dogs and dogs affected with HAC was significantly different $(p=0.019)$. Presence of microalbuminuria and albuminuria in clinically healthy dogs and dogs affected with HAC was significantly different ( $p<0.001)$; significantly higher incidence of microalbuminuria, but significantly lower incidence of albuminuria in clinically healthy dogs compared to dogs affected with HAC. Overall, presence of microalbuminuria or albuminuria was not associated with the status of blood pressure in either clinically healthy dogs or dogs with HAC $(p=0.141)$.

\section{Comparison of systemic blood pressure, presences of microalbuminuria/albuminuria and results of $\mathrm{ACTH}$ stimulation test between dogs affected with PDH and $\mathrm{ADH}$}

The mean systemic blood pressure was significantly different between dogs affected with $\mathrm{PDH}$ and $\mathrm{ADH}$ $(p=0.004)$. Incidence of hypertension in dogs affected with $\mathrm{PDH}$ and $\mathrm{ADH}$ was significantly different $(p=0.017)$. Presence of microalbuminuria and albuminuria in dogs affected with $\mathrm{PDH}$ and ADH was significantly different $(p=0.007)$. However, albuminuria was not associated with the status of blood pressure $(p=0.306)$.

Both pre- and post-ACTH cortisol concentrations between dogs affected with $\mathrm{PDH}$ and $\mathrm{ADH}$ were significantly different $(p<0.001)$. No correlation between systemic blood pressure in either pre-ACTH $(r=0.095$, $p=0.479)$ or post-ACTH cortisol concentrations $(r=0.130, p=0.326)$ was found.

Overall, the incidence of hypertension was highest in dogs with $\mathrm{ADH}$, followed by dogs with $\mathrm{PDH}$, with the lowest levels in clinically healthy dogs $(p=0.019)$; incidences of albuminuria followed the same pattern: highest incidence in dogs with $\mathrm{ADH}$, and lowest level in clinically healthy dogs $(p<0.001)$; but microalbuminuria showed a different pattern: clinically healthy dogs had highest incidence and dogs with ADH had lowest incidence.

\section{Discussion}

The incidence of hypertension in dogs affected with HAC has been reported to range from $52.6(n=10 / 16)$ 
to $86.1 \%(n=31 / 36)[3,4]$. In this study, the incidence of hypertension in dogs with HAC was $46.7 \%$, which was significantly higher than the $12 \%$ incidence observed in clinically healthy dogs, supporting previous observations that dogs affected by HAC tend to have hypertension $[3,4]$. However, the incidence of hypertension in dogs affected with HAC was lower than that reported in previous studies. Different methods of blood pressure measurements, definition of hypertension, and case numbers may explain lower incidence in this study. The degree of hypertension is also positively correlated with the duration of the condition rather than with cortisol concentrations $[11,18]$. In this study, the condition of hyperadrenocorticism was newly diagnosed. Systemic blood pressure was measured at the initial diagnosis. A higher incidence of hypertension might be seen at later stages of the condition.

In this study, higher systemic blood pressure and higher incidence of hypertension was associated with ADH compared to PDH. This finding has not been reported in dogs affected with hyperadrenocorticism. However, no correlation between the cortisol concentrations and systemic blood pressure was found. Similar results were also reported in human patients with Cushing's syndrome $[11,18]$. The concentration of cortisol alone may not cause hypertension. Many factors have been found to be involved in development of hypertension in human patients with Cushing's syndrome, including the duration of hypercortisolaemia, impaired microvascular reactivity, endothelial dysfunctions, and hyperaldosteronemia- associated arterial wall stiffness and fibrosis $[11,18,19,29]$. Higher plasma aldosterone concentrations were reported in dogs affected with ADH than in dogs with PDH $[6,30]$. Nevertheless, aldosterone was not involved in the development of hypertension in dogs with PDH [30]. Further study regarding association between vascular stiffness, myocardial reactivity and systemic blood pressure in dogs affected with either PDH or ADH is warranted.

Incidence of microalbuminuria in clinically healthy dogs in this study (45\%) was similar to previous studies, ranging from 19 to $52 \%$ [31,32]. Surprisingly, a significantly lower incidence of microalbuminuria, but a higher incidence of albuminuria was observed in dogs affected with HAC compared to clinically healthy dogs. Microalbuminuria is a well-known risk factor associated with deteriorated renal and cardiovascular diseases in humans $[12,14,17,20,21]$. It is also associated with survival time in chronic renal failure [22]. Nonetheless, microalbuminuria was also found to be associated with various underlying systemic diseases, such as neoplasia, infectious diseases, and immune or inflammatory diseases $[24,32]$. Due to the high incidence of microalbuminuria in clinically healthy dogs and the association of this condition with various systemic disorders, the microalbuminuria test may be more useful to screen for subclinical disorders than to detect specific diseases.

In this study, incidence of hypertension and albuminuria was significantly higher in dogs affected with $\mathrm{ADH}$ compared to PDH. This finding has not been reported in dogs affected with HAC. The correlation between systemic blood pressure and albuminuria would be anticipated in dogs with HAC, however, this was not supported in this study. Hypertension is not the only factor associated with presence of albuminuria. Increased urinary albumin excretion rate is also linked to dyslepidaemia, reduced insulin sensitivity, impaired endothelial function and hyper-coagulative status [7]. These complications are commonly seen in both human and canine patients with Cushing's syndrome $[1,16,18,19]$. Glomerular changes and subsequently, increases in urinary protein excretion were found with chronic glucocorticoid use [33,34]. These may explain the disassociation between albuminuria and blood pressure in these dogs.

The cause of albuminuria in dogs affected with HAC can not be answered in this study. Nevertheless, longterm monitoring of albuminuria in terms of treatment and survival time is needed to clarify the importance of albuminuria and HAC in dogs.

\section{Conclusions}

Incidences of hypertension and albuminuria, but not microalbuminuria, were highest in dogs affected with $\mathrm{ADH}$, followed by dogs with $\mathrm{PDH}$, with the lowest levels observed in clinically healthy dogs. However, the presence of albuminuria was not correlated with systemic blood pressure.

\section{Acknowledgements}

The authors also thank Dr. Kai-Chung Chang at the Consulting Center for Statistics and Bioinformatics, College of Bio-Resources and Agriculture, National Taiwan University for assistance with the statistical analyses.

\section{Author details}

${ }^{1}$ Azu Clinic for Animals, No. 92, Section 1, Kin-Shan South Road, Taipei 100, Taiwan. ${ }^{2}$ Institute of Clinical Veterinary Science, National Taiwan University, No. 1, Section 4, Roosevelt Road, Taipei 100, Taiwan.

\section{Authors' contributions}

$\mathrm{YHL}$ participated in the designs of the study and carried out the recruitment of cases of hyperadrenocorticism. She also drafted the manuscript. TYH performed measurement of systemic blood pressure, carried out the analysis of urinary albumin and creatinine, and the recruitment of clinically healthy dogs as control in this study. HPH participated in the designs of the study, carried out the recruitment of cases of hyperadrenocorticism and performed the statistical analysis and the manuscript writing. All authors read and approved the final manuscript.

\section{Competing interests}

The authors declare that they have no competing interests.

Received: 16 March 2010 Accepted: 12 November 2010 Published: 12 November 2010 


\section{References}

1. Feldman EC, Nelson RW: Canine hyperadrenocorticism. Canine and Feline Endocrinology and Reproduction. 3 edition. St Louis: Saunders; 2004, 253-353.

2. Hurley KJ, Vaden SL: Evaluation of urine protein content in dogs with pituitary-dependent hyperadrenocorticism. J Am Vet Med Assoc 1998, 212:369-373.

3. Novellas R, de Gopegui RR, Espada Y: Determination of renal vascular resistance in dogs with diabetes mellitus and hyperadrenocorticism. Vet Rec 2008, 163:592-596.

4. Ortega TM, Feldman EC, Nelson RW, Willits N, Cowgill LD: Systemic arterial blood pressure and urine protein/creatinine ratio in dogs with hyperadrenocorticism. J Am Vet Med Assoc 1996, 209:1724-1729.

5. De Martin M, Giraldi FP, Cavagnini F: Cushing's Disease. Pituitary 2006, 9:279-287.

6. Javadi S, Kooistra HS, Mol JA, Rijnberk A, Boer P, Boer WH: Plasma aldosterone concentrations and plasma renin activity in healthy dogs and dogs with hyperadrenocorticism. Vet Rec 2003, 153:521-525.

7. Karalliedde J, Viberti G: Microalbuminuria and cardiovascular risk. Am J Hypertens 2004, 17:986-993.

8. Koh JM, Kim JY, Chung YE, Park JY, Shong YK, Hong SK, Kim GS, Lee KU: Increased urinary albumin excretion in Cushing's syndrome: remission after correction of hypercortisolaemia. Clin Endocrinol 2000, 52:349-353.

9. Magiakou MA, Smyrnaki P, Chrusos GP: Hypertension in Cushing's syndrome. Best Pract Res Clin Endocrinol Metab 2006, 20:467-482.

10. Sartori M, Calò LA, Mascagna V, Realdi A, Macchini A, Ciccariello L, De Toni R, Cattelan F, Pessina AC, Semplicini A: Aldersterone and refractory hypertension: a prospective cohort study. Am J Hypertens 2006, 19:373-379.

11. Tsuiki M, Tanabe A, Takagi S, Naruse M, Takano K: Cardiovascular risks and their long-term clinical outcome in patients with subclinical Cushing's syndrome. Endocr J 2008, 55:737-745.

12. Bigazzi R, Bianchi S, Baldari D, Campese VM, Campese VM: Microalbuminuria predicts cardiovascular events and renal insufficiency in patients with essential hypertension. J Hypertens 1998, 16:1325-1333.

13. De Jong PE, Gansevoort RT: Focus on microalbuminuria to improve cardiac and renal protection. Nephron 2009, 111:c204-210.

14. Janssen SF, Twickler TB, Jublanc C, Cramer MJ, Bruckert E: Patients with the metabolic syndrome and a disturbed cortisol balance display more microalbuminuria. Diabetes \& Vascular Disease Research 2008, 5:54-58.

15. Mogensen CE: Microalbuminuria and hypertension with focus on type 1 and 2 diabetes. J Intern Med 2003, 254:45-66.

16. Nogueira ADR, Fernandes AS, Coutinho ESF, Salles GF, Muxfeld ES, Bloch KV: Factors associated with microalbuminuria in resistant hypertension. Int J Cardiol 2007, 121:86-87

17. Volpe M: Microalbuminuria screening in patients with hypertension: recommendations for clinical practice. Int J Clin Pract 2008, 62:97-108.

18. Mancini T, Kola B, Mantero F, Boscaro M, Arnoldi G: High cardiovascular risk in patients with Cushing's syndrome according to 1999 WHO/ISH guidelines. Clin Endocrinol 2004, 61:768-777.

19. Prázný M, Ježková J, Horová E, Lazárová V, Hána V, Kvasnička J, Pecen L, Marek J, Škrha J, Kršek M: Impaired microvascular reactivity and endothelial function in patients with Cushing's syndrome: influence of arterial hypertension. Physiol Res 2008, 57:13-22

20. Futrakul N, Sridama V, Futrakul P: Microalbuminuria-a biomarker of renal microvascular disease. Ren Fail 2009, 31:140-143.

21. Vyssoulis P, Karpanou EA, Michaelidis AP, Barbetseas JD, Kyvelou SM Gialernios TP, Cokkinos DV, Stefanadis Cl: Microalbuminuria and global myocardial function in patients with essential hypertension. Int J Cardio 2008, 126:268-272

22. Syme HM, Markwell PJ, Pfeiffer D, Elliott J: Survival of cats with naturally occurring chronic renal failure is related to severity of proteinuria. J Vet Intern Med 2006, 20:528-535.

23. Whittemore JC, Miyoshi Z, Jensen WA, Radecki SV, Lappin MR: Association of microalbuminuria and the urine albumin-to-creatinine ratio with systemic disease in cats 2007. J Am Vet Med Assoc 2007, 230:1165-1169.

24. Whittemore JC, Gill VL, Jensen WA, Radecki SV, Lappin MR: Evaluation of the association between microalbuminuria and the urine albumincreatinine ratio and systemic disease in dogs. J Am Vet Med Assoc 2006, 229:958-963.
25. Lien YH, Huang HP: Use of ketoconazole to treat dogs with pituitarydependent hyperadrenocorticism: 48 cases (1994-2007). J Am Vet Med Assoc 2008, 233:1896-1901.

26. Barthez PY, Nyland TG, Feldman EC: Ultrasonographic evaluation of the adrenal glands in dogs. J Am Vet Med Assoc 1995, 207:1180-1183.

27. Hoerauf $A$, Reusch C: Ultrasonographic characteristics of both adrenal glands in 15 dogs with functional adrenocortical tumors. J Am Anim Hosp Assoc 1999, 35:193-199.

28. Hsiang TY, Lien YH, Huang HP: Indirect measurement of systemic blood pressure in conscious dogs in a clinical setting. J Vet Med Sci 2008, 70:449-453.

29. Štrauch B, Petrák O, Wichterle D, Zelinka T, Holaj R, Widimský J Jr: Increased arterial wall stiffness in primary aldosteronism in comparison with essential hypertension. Am J Hypertens 2006, 19:909-914.

30. Goy-Thollot I, Péchereau D, Kéroack S, Dezempte JC, Bonnet JM: Investigation of the role of aldosterone in hypertension association with spontaenous pituitary-dependent hyperadrenocorticism in dogs. J Small Anim Pract 2002, 43:489-492.

31. Jensen WA, Grauer GF, Andrew J, Simpson D: Prevalence of microalbuminuria in dogs. J Vet Intern Med 2001, 15:300.

32. Pressler BM, Vaden SL, Jensen WA, Simpson D: Prevalence of microalbuminuria in dogs evaluated at a referral veterinary hospital. $J$ Vet Intern Med 2001, 15:300.

33. Schellenberg S, Mettler M, Gentilini F, Portmann R, Glaus TM, Reusch CE: The effects of hydrocortisone on systemic arterial blood pressure and urinary protein excretion in dogs. J Vet Intern Med 2008, 22:273-281.

34. Waters CB, Adams LG, Scott-Moncrieff JC, DeNicola DB, Snyder PW, White MR, Gasparini M: Effects of glucocorticoid therapy on urine protein-to-creatinine ratios and renal morphology in dogs. J Vet Intern Med 1997, 11:172-177.

doi:10.1186/1751-0147-52-61

Cite this article as: Lien et al:: Associations among systemic blood pressure, microalbuminuria and albuminuria in dogs affected with pituitary- and adrenal-dependent hyperadrenocorticism. Acta Veterinaria Scandinavica 2010 52:61.

\section{Submit your next manuscript to BioMed Central and take full advantage of:}

- Convenient online submission

- Thorough peer review

- No space constraints or color figure charges

- Immediate publication on acceptance

- Inclusion in PubMed, CAS, Scopus and Google Scholar

- Research which is freely available for redistribution

Submit your manuscript at www.biomedcentral.com/submit
Biomed Central 